\title{
Signet-ring cell carcinoma of the colon: A case report of a 9-year-old boy
}

\author{
SHAOBO YANG ${ }^{1}$, GONGBAO LIU $^{1}$, SHAN ZHENG $^{1}$, KUIRAN DONG $^{1}$, YANGYANG MA $^{2}$ and XIANMIN XIAO ${ }^{1}$ \\ Departments of ${ }^{1}$ Surgery and ${ }^{2}$ Pathology, Children's Hospital of Fudan University, Minhang, Shanghai 201102, P.R. China
}

Received August 19, 2014; Accepted May 7, 2015

DOI: $10.3892 / 01.2015 .3403$

\begin{abstract}
Colorectal carcinomas are common in adults; however, they rarely occur in children. The present study reported the case of a colorectal carcinoma diagnosed as signet-ring cell carcinoma in a 9-year-old boy. The patient presented with acute intestinal obstruction symptoms, which persisted following 3 days of conservative treatment. The diagnosis was determined by emergency laparotomy and a mass was identified in the transverse colon proximal to the splenic flexure. Histological examination of the mass revealed signet-ring cell carcinoma. In addition, the present study aimed to discuss the diagnostic establishment and treatment of this rare disease in children. Due to the high mortality rate caused by delays in accurate diagnoses, signet-ring cell carcinoma in children promotes a specific diagnostic and surgical challenge.
\end{abstract}

\section{Introduction}

Colorectal carcinoma is the third most prevalent type of cancer in adults, which has an incidence rate that increases with age (1). Colorectal carcinoma rarely occurs in children, with an incidence rate of between 1 and 8 cases per million children worldwide (2). Therefore, pediatricians and pediatric surgeons are not familiar with colorectal carcinoma in children and its clinical manifestation are different from those in adults (3). Colorectal carcinoma may be detected by digital rectal examination, sigmoidoscopy or colonoscopy. Furthermore, contrast studies, ultrasonography and computed tomography are essential procedures for determining the extent of the disease. Postoperative histological examination is considered the gold standard for diagnosis. Colorectal carcinoma in children may occur at any age, however, the majority of cases are identified in older children; $70-95 \%$ of cases occur in children $>10$ years of age $(2,4-6)$. The present study reports the case of colorectal

Correspondence to: Dr Xianmin Xiao, Department of Surgery, Children's Hospital of Fudan University, 399 Wanyuan Road, Minhang, Shanghai 201102, P.R. China

E-mail: xianminxiao@163.com

Key words: colorectal carcinoma, signet-ring cell carcinoma, intestinal obstruction, children carcinoma, diagnosed as signet-ring cell carcinoma, in a 9-year-old boy.

\section{Case report}

A 9-year-old boy with no history of medical illness was presented to the emergency department of the Children's Hospital of Fudan University (Shanghai, China) with primary complaints of abdominal pain, which had persisted for 1 day. This was preceded by intermittent vomiting 5 days prior to admission, which was treated as an intestinal obstruction in a local health center. Family history revealed nothing of significance. On admission, the patient appeared irritable and in distress due to pain. Physical examination revealed abdominal distension and tenderness in the upper abdomen with positive bowel sounds; however, muscle tension was not palpated.

Initial laboratory tests revealed the following: White cell count, $6,900 / \mu 1$ with $62.8 \%$ neutrophils; C-reactive protein, $66 \mathrm{mg} / \mathrm{l}$; hemoglobin, $116.0 \mathrm{~g} / \mathrm{l}$; urea, $3.10 \mathrm{mmol} / \mathrm{l}$; serum creatinine, $28.0 \mu \mathrm{mol} / \mathrm{l}$; sodium, $136 \mathrm{mmol} / \mathrm{l}$; potassium, $3.7 \mathrm{mmol} / \mathrm{l}$; glutamic-pyruvic transaminase, $5 \mathrm{IU} / \mathrm{l}$; glutamic oxalacetic transaminase, $11 \mathrm{IU} / 1$; and albumin, $41 \mathrm{~g} / 1$. Urinalysis results were normal. Abdomen X-ray revealed an incomplete obstruction of the upper section of the colon.

The patient was treated with a third-generation cephalosporin and fluid infusion, accompanied by fasting and gastrointestinal decompression. Following 3 days of treatment, the intestinal obstruction was not relieved. A further abdominal enhanced computed tomography (CT) scan depicted significant stenosis in the transverse colon proximal to the splenic flexure, with local bowel wall thickening; therefore, an occupying lesion could not be excluded (Fig. 1). An emergency laparotomy was performed in order to diagnose the mass. During surgery, a mass measuring $4 \times 4 \mathrm{~cm}$ was identified in the transverse colon proximal to the splenic flexure (Fig. 2), which formed a near complete obstruction of the enteric cavity. The proximate colon was notably expanded (maximum diameter, $\sim 10 \mathrm{~cm}$ ) and the distant colon was narrow (diameter, $\sim 4 \mathrm{~cm}$ ). Following proximate bowel decompression, the retroperitoneal lymphaden and liver were explored, although no abnormalities were observed. The transverse colon where the mass was located was excised $(5 \mathrm{~cm}$ surgical margin around the tumor) and a single lumen stoma was performed at the proximate end. Due to insufficient bowel preparation for the emergency operation, I-stage anastomosis was at great risk of 
postoperative infection and leaking. Lymph node dissection was not performed because a diagnosis of colorectal carcinoma had not been considered. Histological examination of the mass revealed signet-ring cell carcinoma, encroaching the intestinal canal (Fig. 3). Further laboratory tests revealed the following: Cancer antigen (CA)125 expression, $85.4 \mathrm{IU} / \mathrm{ml}$ (reference, $<35 \mathrm{IU} / \mathrm{ml}$ ); CA19-9 expression, $12.9 \mathrm{U} / \mathrm{ml}$ (reference, $<37 \mathrm{U} / \mathrm{ml}$ ); and carcinoembryonic antigen (CEA) expression, $0.9 \mathrm{ng} / \mathrm{ml}$ (reference, $<5.0 \mathrm{ng} / \mathrm{ml}$ ). Abdomen ultrasonography as well as cephalic, chest and abdomen CT scans and bone scintigraphy demonstrated no metastases. The stage of tumor was determined to be Tumor ${ }_{3}$ Node $_{x}$ Metastasis $_{0}$ and Duke's stage B or C (7). Lymphadenectomy and postoperative adjuvant chemotherapy were planned to be perform. However, the patient's parents objected to therapy and the patient was discharged 2 weeks following surgery.

The patient underwent chemotherapy 7 times in another hospital for one year following surgery. Following one year, the patient returned to the Children's Hospital of Fudan University in order to close the stoma. The patient underwent re-operation, during which a mesenteric lymph node metastasis was identified. An abdominal CT follow-up revealed mesenteric lymph node metastasis and CEA expression had risen to $87.0 \mathrm{ng} / \mathrm{ml}$ (reference, $<5.0 \mathrm{ng} / \mathrm{ml}$ ). The patient succumbed to the disease at 2 weeks following discharge from hospital; cephalic CT scans revealed metastases.

\section{Discussion}

Signet-ring cell carcinoma, a type of colorectal carcinoma, is scarcely diagnosed in children. Sultan et al (3) reported that signet-ring cell carcinoma accounted for $18 \%$ of colorectal carcinomas in children and adolescents.

Adult cases of colorectal cancer usually present with manifestations that include abdominal pain, hematochezia, difficult defecation and attenuated stool (8). However, in children it is difficult to decipher their primary complaint and neoplastic lesions are generally more severe when examined by a physician due to parents' negligence. Patients commonly present with advanced intestinal obstruction symptoms on admission, as in the current case report. Early symptoms of the current patient included intermittent vomiting and abdominal pain, and the diagnosis was determined by an emergency surgery, during which the intestinal obstruction became obvious. Therefore, the possibility of a tumor should be considered for every child admitted to hospital with intestinal obstruction symptoms, incurable abdominal pain or changed bowel evacuation habits. B-mode ultrasound or enhanced CT contribute to the early identification of colorectal carcinoma; however, the principal method of colorectal carcinoma diagnosis is colonoscopy, with an accuracy of 90-95\% (9). Final diagnosis is dependent on pathological examination. CEA is a reference index of auxiliary diagnosis and relapse monitoring, though it has no tumor specificity.

Signet-ring cell carcinoma has an aggressive clinical course and a high lymphatic metastasis rate; it is known to metastasize early and 60-100\% of children are at advanced stage on admission, stage $\mathrm{C}$ or D according to Dukes staging (7). Radical surgery is the preferred treatment for signet-ring cell carcinoma, even for patients of advanced stages. In addition, signet-ring cell

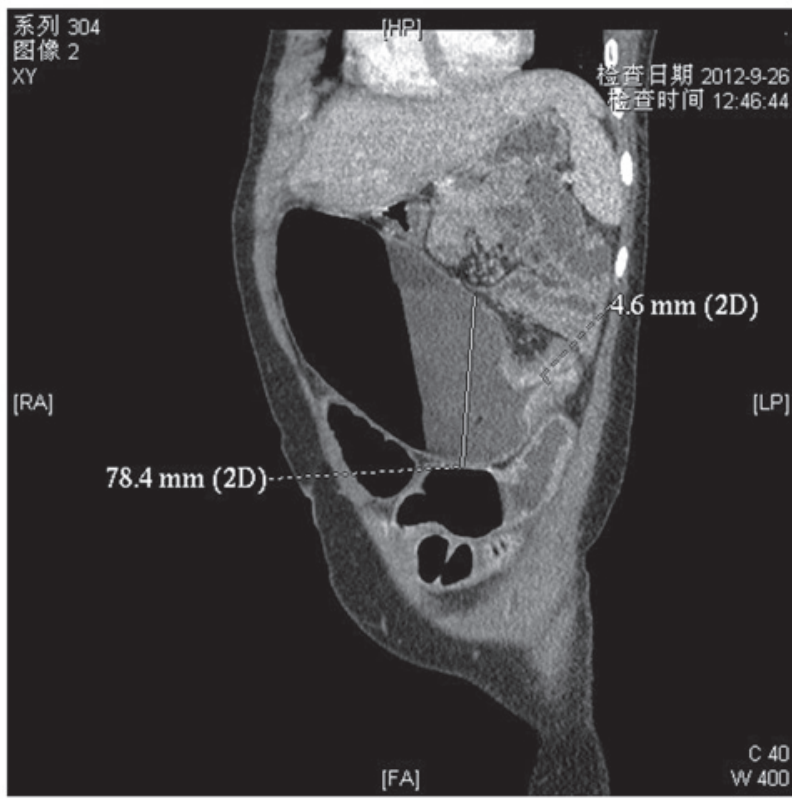

Figure 1. Abdomen enhanced computed tomography image depicting a marked stenosis in the transverse colon proximal to the splenic flexure with local bowel wall thickening.

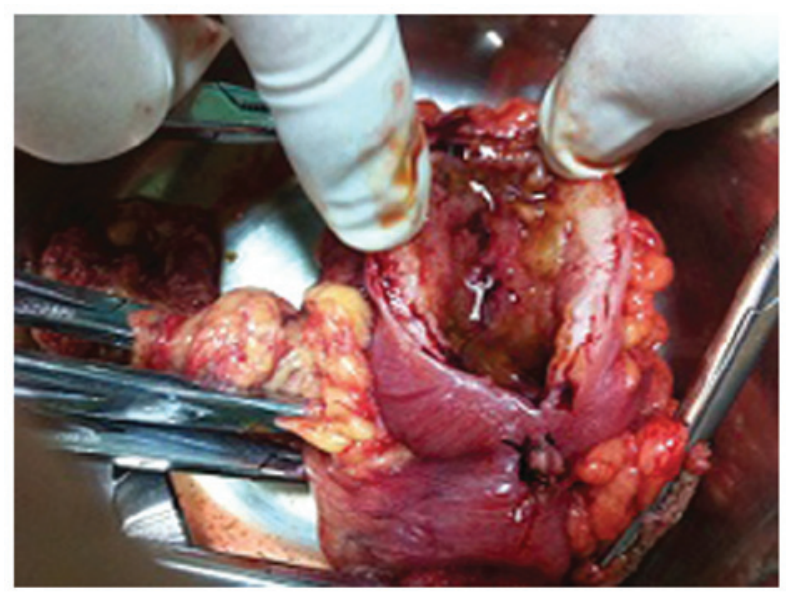

Figure 2. During surgery, a mass, measuring $4 \times 4 \mathrm{~cm}$, was identified in the transverse colon proximal to the splenic flexure.

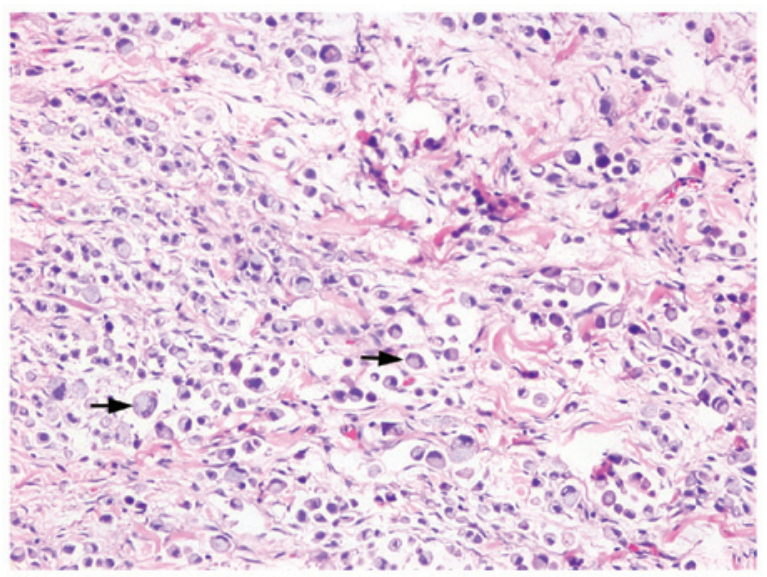

Figure 3. Histological examination of the mass located during surgery revealed allotype tumor cells; certain cells were identified to be signet-ring cells (arrows; magnification, x200). 
carcinoma first metastasizes to para-intestinal lymph nodes and the range of metastasis is $\sim 10 \mathrm{~cm}$ from the tumor (10).

As signet-ring cell carcinoma rarely occurs in children, frozen pathological examinations should be performed for highly suspect malignant tumors. In addition, the intestinal incisal edge should be $>10 \mathrm{~cm}$ away from the tumor at initial surgery in order to help prevent metastasis. As it is rare for signet-ring cell carcinoma to be identified in patients with intestinal obstruction manifestations during surgery, preoperative overall examinations and bowel preparation are insufficient. Therefore it is a dilemma whether to choose palliative or radical resection when tumors are discovered during surgery. In the present case study, palliative fistulation was performed at the initial exploratory laparotomy due to insufficient preoperative bowel preparation and high risk of primary inosculation.

Postoperative chemotherapy is indispensable to colorectal carcinoma; at present, adjuvant chemotherapy with 5-fluorouracil is generally accepted, in particular for stage III or IV patients, as it has been found to reduce the rate of relapse and increase survival rates (11). Postoperative radiotherapy is unable to increase the survival rate of patients with tumors that are difficult to completely resect; however, palliative radiotherapy may effectively relieve the clinical symptoms of metastasized lesions (12).

In conclusion, colorectal carcinomas in children are rare and provide a lack of typical clinical symptoms and radiological data, as demonstrated in the present case study. In addition, the clinical symptoms are often undervalued by parents and doctors; this therefore results in poor prognosis of advanced stage tumors, when diagnosed. It is therefore suggested that malignancies should be considered by pediatricians and pediatric surgeons for differential diagnosis when patients present with intestinal obstruction symptoms, incurable abdominal pain or changed bowel evacuation habits, as early diagnosis and treatment decreases the mortality rate of patients. This is of particular importance for signet-ring cell carcinoma of the colon or rectum, as it has a poor prognosis at advanced stages.

\section{References}

1. Jemal A, Siegel R, Ward E, Hao Y, Xu J and Thun MJ: Cancer statistics, 2009. CA Cancer J Clin 59: 225-249, 2009.

2. Yang R, Cheung MC, Zhuge Y, Armstrong C, Koniaris LG and Sola JE: Primary solid tumors of the colon and rectum in the pediatric patient: A review of 270 cases. J Surg Res 161: 209-216, 2010.

3. Sultan I, Rodriguez-Galindo C, El-Taani H, et al: Distinct features of colorectal cancer in children and adolescents: A population-based study of 159 cases. Cancer 116: 758-765, 2010.

4. Brown RA, Rode H, Millar AJ, Sinclair-Smith C and Cywes S: Colorectal carcinoma in children. J Pediatr Surg 27: 919-921, 1992.

5. Karnak I, Ciftci AO, Senocak ME and Büyükpamukçu N: Colorectal carcinoma in children. J Pediatr Surg 34: 1499-1504, 1999.

6. Vastyan AM, Walker J, Pintér AB, Gerrard M and Kajtar P: Colorectal carcinoma in children and adolescents - a report of seven cases. Eur J Pediatr Surg 11: 338-341, 2001.

7. Radhakrishnan CN and Bruce J: Colorectal cancers in children without any predisposing factors. A report of eight cases and review of the literature. Eur J Pediatr Surg 13: 66-68, 2003.

8. Hill DA, Furman WL, Billups CA, et al: Colorectal carcinoma in childhood and adolescence: A clinicopathologic review. J Clin Oncol 25: 5808-5814, 2007.

9. Beck DE: Colorectal cancer screening. Clin Colorectal Surg 14: 115, 2001.

10. Schumacher P, Dineen S, Barnett C Jr, Fleming J and Anthony T: The metastatic lymph node ratio predicts survival in colon cancer. Am J Surg 194: 827-832; discussion, 831-832, 2007.

11. Goldberg RM: N9741: A phase III study comparing irinotecan to oxaliplatin-containing regimens in advanced colorectal cancer. Clin Colorectal Cancer 2: 81, 2002.

12. Labianca R, Beretta G, Gatta G, De Braud F and Wils J: Colon cancer. Crit Rev Oncol Hematol 51: 145-170, 2004. 